\title{
Fabrication and Characterization of Tin Oxide Inverse Opal by Template Method
}

\author{
Jinquan Wang ${ }^{\mathrm{a}, \mathrm{b}}$, Wu Xinhua ${ }^{\mathrm{a}}$, Wu Yuanyuan ${ }^{\mathrm{a}}$, Li Jie ${ }^{\mathrm{a}}$, Yuan Saisai ${ }^{\mathrm{a}}$, Ming Zhang ${ }^{\mathrm{a},{ }^{\star}}$, Xiaobing Chen ${ }^{\mathrm{a},{ }^{\star}}$ \\ ${ }^{a}$ College of Chemistry and Chemical Engineering, Yangzhou University, Yangzhou, 225002 Jiangsu, P. R. China \\ ${ }^{\mathrm{b}}$ Huaiyin Institue of Technology, Huaiyin, 223003, Jiangsu, P.R.China
}

*Corresponding Author: 1xyzhangm@yzu.edu.cn, xbchen@yzu.edu.cn

\begin{abstract}
.
In this study, Tin Oxide $\left(\mathrm{SnO}_{2}\right)$ inverse opal was fabricated by infiltrating $\mathrm{SnO}_{2}$ sol-gel precursor solution into Poly Styrene (PS) spheres crystal template which was generated via evaporative deposition self-assembling, then was heat treating at $500^{\circ} \mathrm{C}$ for two hours in controlled atmosphere box furnaces. PS spheres crystal template was characterized by Scanning electron microscope and Ultraviolet-visible-near infrared absorption spectrometer. The $\mathrm{SnO}_{2}$ inverse opal was characterized by Scanning electron microscope, Energy Diffraction Spectrum, X Ray Diffraction. The large-area, highly ordered $\mathrm{SnO}_{2}$ inverse opal was achieved in this paper.
\end{abstract}

Keywords: Opal, Tin Oxide, Inverse opal, Template method

\section{Introduction}

As an important gas sensitive material, $\mathrm{SnO}_{2}$ has been found extensive applications in the past, and is still a hot topic of the research regarding gas sensitive materials. This is because $\mathrm{SnO}_{2}$ has indispensable advantages such as high sensitivity, low cost and good availability, and high thermal ability. The larger superficial area of $\mathrm{SnO}_{2}$ is achieved, the higher sensitivity shows. Several groups have fabricated and studied $\mathrm{SnO}_{2}$ inverse opal ${ }^{[1][2][3]}$, but they could not achieve large-area, high ordered $\mathrm{SnO}_{2}$ inverse opal. In this paper, the large-area, highly ordered $\mathrm{SnO}_{2}$ inverse opal was achieved by template method ${ }^{[4][5]}$.

\section{Experimental Section}

PS opal fabrication

Polystyrene (PS) spheres with diameter about 270nm and a standard deviation of less than 5\% were prepared by using the emulsifier-free emulsion polymerization technique ${ }^{[6][7]}$. The PS spheres produced were cleaned by consecutive centrifuging, decanting, and re-dispersing, first in water (three times) and then in ethanol (three times).

Glass microscope slides were cleaned in freshly prepared Piranha solution $\left(\mathrm{H}_{2} \mathrm{SO}_{4} / \mathrm{H}_{2} \mathrm{O}_{2}, 3: 1\right)$, and rinsed copiously first with deionized water and then with ethanol. 270nm PS sphere dispersions $(10 \mathrm{~mL})$ with typical concentrations about $0.1 \mathrm{wt} \%$ were placed in a cylindrical clear glass vial. The cleaned glass microscope slide was carefully centered in the cylindrical clear glass vial and vertically fixed with two small paper clips. Then the glass vial was taken into oven where the temperature was $55{ }^{\circ} \mathrm{C}$. After about 20 hours, the liquid volatilized over, the opal on the glass microscope was achieved.

$\mathrm{SnO}_{2}$ inverse opal fabrication:

Mixed solution of $40 \mathrm{ml} \mathrm{SnCl}_{4}$ solution $(0.13 \mathrm{M})$ and $0.4 \mathrm{~g}$ citric acid were stirred and heated to $50{ }^{\circ} \mathrm{C}$, ammonia water was added into the mixed solution until the $\mathrm{pH}$ of mixed solution was about $1.5-2$, sediment came out at the same time ${ }^{[8]}$. The sediment was cleaned by consecutive centrifuging, decanting, and re-dispersing, until $\mathrm{Cl}^{-}$in upper clear water could not be checked out with $\mathrm{AgNO}_{3}$ solution. The sediment solution was heated to $50{ }^{\circ} \mathrm{C}$ and dissolved with oxalic acid saturated solution as $\mathrm{pH}$ was about 1.5-2, 
then was diluted with deionized water to $60 \mathrm{ml}$.

The opal on the glass microscope was immersed in $\mathrm{SnO}_{2}$ precursor solution, then taken into vacuuming box and vacuumed 15 minutes, then taken into oven where the temperature was $55{ }^{\circ} \mathrm{C}$ to dry for 30 minutes. The operation above was repeated three times. After those operations, the infiltrated opal was heated from room temperature to $500{ }^{\circ} \mathrm{C}$ for 140 minutes, maintained at $500{ }^{\circ} \mathrm{C}$ for 2 hours, then cooled down to room temperature for 15 hours.

\section{Result and discussion}

A S-4800 II FESEM was used for direct characterization of the opal. Figure 1(a) shows typical SEM surface images of 270nm PS colloidal crystal which exhibits ordered close-packed arrangement of PS spheres. Figure 1(b) shows typical SEM cross-section images of $270 \mathrm{~nm}$ PS colloidal crystals which exhibits inner lattice structure and some vacancies on the surface ${ }^{[9]}$.
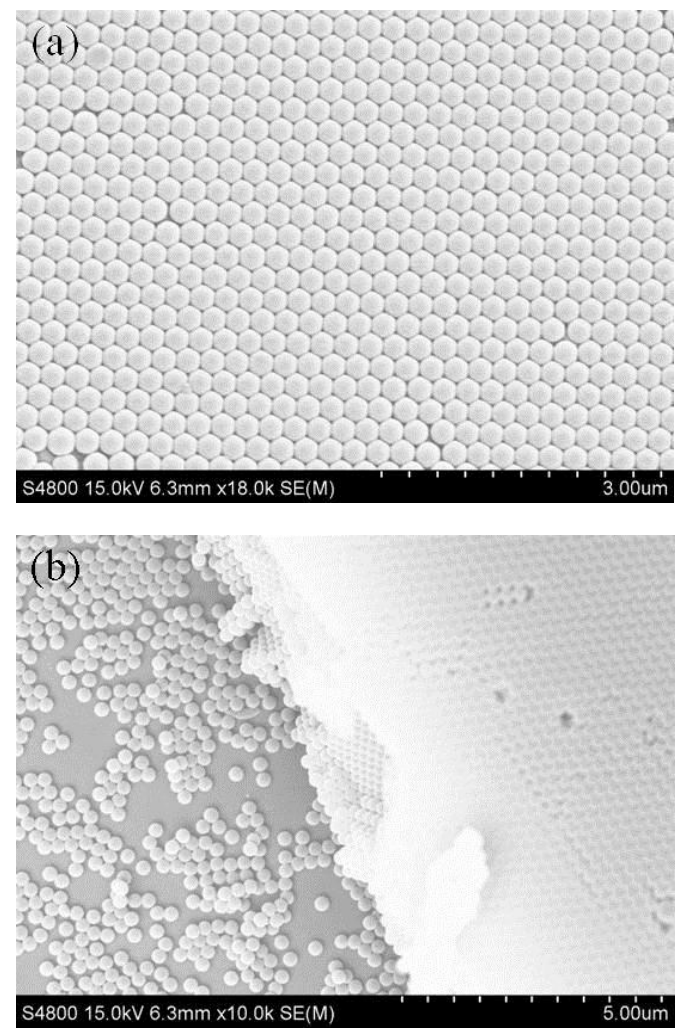

Figure 1. Scanning electron microscope image of $270 \mathrm{~nm}$ PS colloidal crystal: (a) top view; (b) cross-section view.

In order to further research the inner lattice structure, Cary 500 (UV-NIR) was used for characterization of the opal. Figure 2 shows that the 270nm PS colloidal crystal has good lattice structure. The photonic band gap position is in $620 \mathrm{~nm}$ that is corresponding to Bragg's law ${ }^{[10]}$ :

$$
\lambda_{\max }=2 \mathrm{~d}_{111} \sqrt{n_{\text {avg }}{ }^{2}-\sin ^{2} \theta}
$$

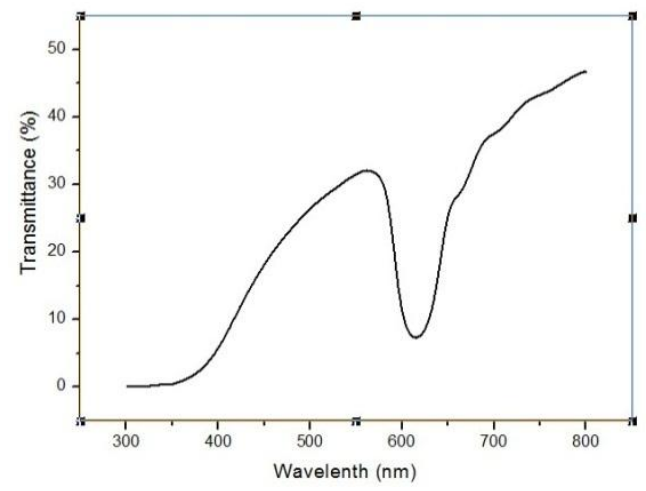

Figure 2.Optical transmittance spectra of $270 \mathrm{~nm}$ PS colloidal crystal.

A S-4800 II FESEM was used for direct characterization of the inverse opal of $\mathrm{SnO}_{2}$. Figure 3 (a) shows that the hundreds of square millimeters of $\mathrm{SnO}_{2}$ inverse opal could be achieved, but some defects can be checked from this picture, some of the defects were the photocopy of the PS spheres opal defects, some of the defects such as over-layer formation was in the process of excessive infiltration of $\mathrm{SnO}_{2}$ precursor solution. The inlaying picture is the higher power of figure3 (a) which shows three holes under every surface hole. Figure 3 (b) exhibits the cross-section of $\mathrm{SnO}_{2}$ inverse opal which has perfect ordered inner structure.
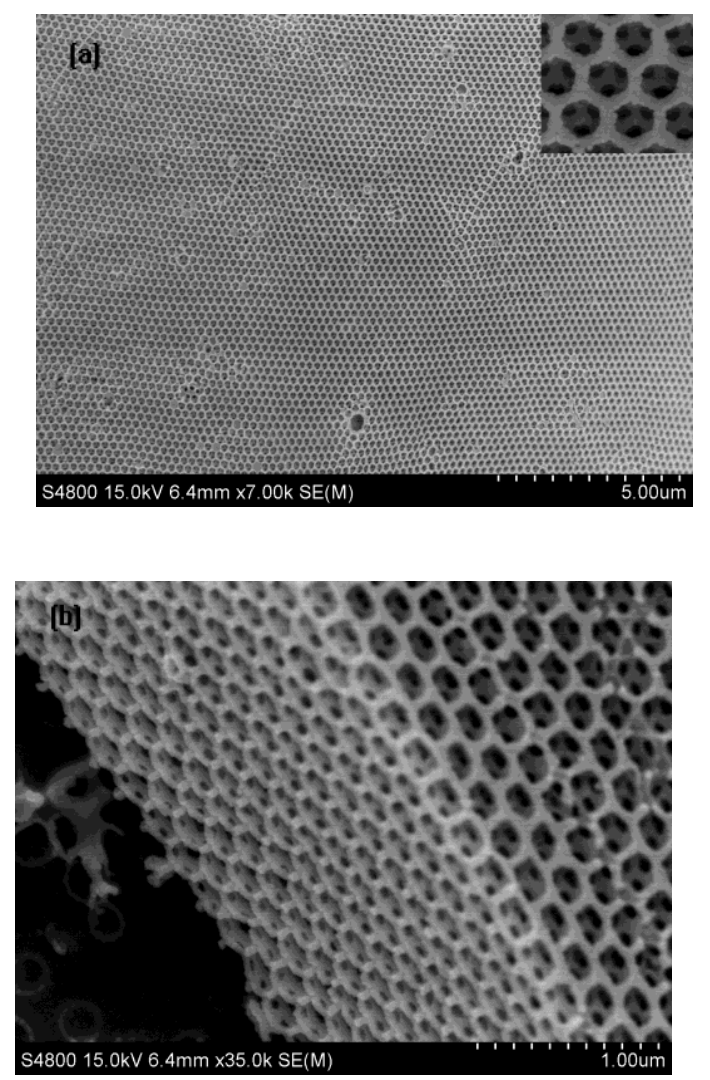
Fig 3.Scanning electron microscope image of $\mathrm{SnO}_{2}$ inverse opal film based on $270 \mathrm{~nm}$ PS colloidal crystal: (a) top view; (b) cross-section view.

In order to further study the $\mathrm{SnO}_{2}$ inverse opal, the EDS was used to detect the element of $\mathrm{SnO}_{2}$ inverse opal. XRD was used to detect the crystal structure of $\mathrm{SnO}_{2}$ inverse opal. Figure 4 exhibits the different elements of the glass microscope slides which clearly shows Tin element existing. Figure 5 is the crystal structure comparing between $\mathrm{SnO}_{2}$ sol gel (heated at $500{ }^{\circ} \mathrm{C}$ for 2 hours) and inverse opal. It shows that the XRD peak position of $\mathrm{SnO}_{2}$ sol gel and inverse opal are same, but the intensity of $\mathrm{SnO}_{2}$ sol gel is stronger than $\mathrm{SnO}_{2}$ inverse opal.

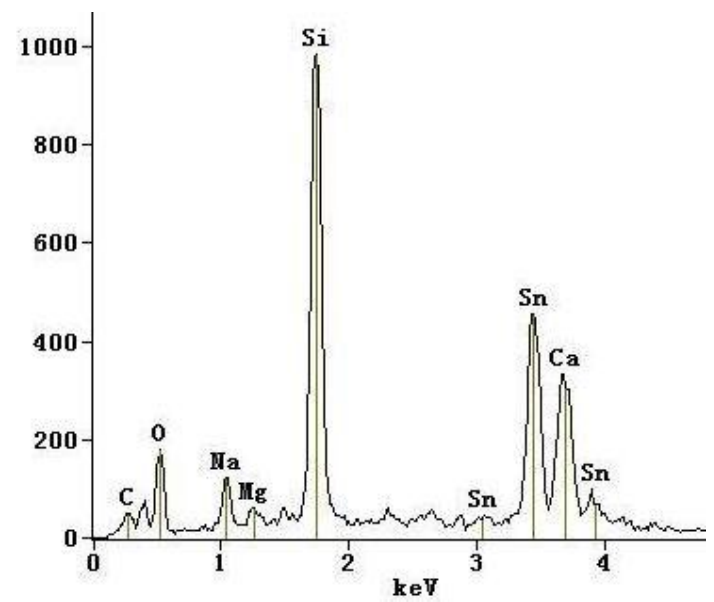

Fig 4.EDS analyses of SnO2 inverse opal.

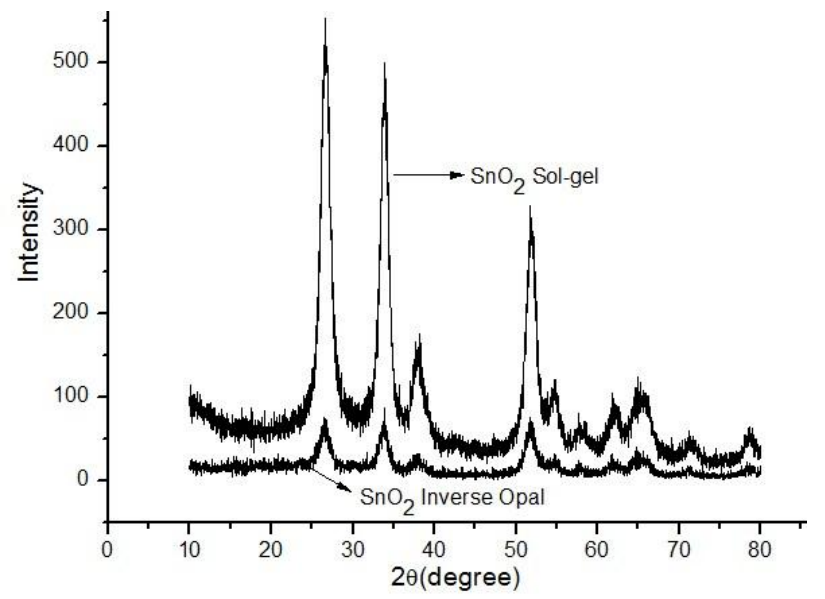

4. Conclusion

$\mathrm{SnO}_{2}$ inverse opal was prepared by the self-assembly technique in combination with sol-gel infiltration method. The $\mathrm{SnO}_{2}$ inverse opal has great specific surface area which can be a catalyst material and gas sensing material. But the fabricated $\mathrm{SnO}_{2}$ inverse opal has some defects such as multi-domains, cracking, over-layers that inhibit application. How to dissolve those defects is an important issue. Our research team is fabricating large-area, highly ordered, crack-free $\mathrm{SnO}_{2}$ inverse opal film and dissolving those problems.

\section{Acknowledgments}

This work was supported by the National Science Foundation of China (No. 51072177) and Jiangsu ordinary university graduate students scientific research innovation project (CXZZ12_0896).

\section{References}

[1] D’Arienzo, M.; Armelao, L.; Cacciamani, A.; Mari, C. M.; Polizzi, S.; Ruffo, R.; Scotti, R.; Testino, A.; Wahba, L.; Morazzoni, F., One-Step Preparation of $\mathrm{SnO}_{2}$ and Pt-Doped $\mathrm{SnO}_{2} \mathrm{As}$ Inverse Opal Thin Films for Gas Sensing. Chemistry of Materials 2010, 22 (13), 4083-4089.

[2] Arsenault, E.; Soheilnia, N.; Ozin, G. A., Periodic Macroporous Nanocrystalline Antimony-Doped Tin Oxide Electrode. Acs Nano 2011, 5 (4), 2984-2988.

[3] Sutti, A.; Baratto, C.; Calestani, G.; Dionigi, C.; Ferroni, M.; Faglia, G.; Sberveglieri, G., Inverse opal gas sensors: $\mathrm{Zn}(\mathrm{II})$-doped tin dioxide systems for low temperature detection of pollutant gases. Sensors and Actuators B: Chemical 2008, 130 (1), 567-573.

[4] Wang, A.; Chen, S.-L.; Dong, P.; Zhou, Z., Preparation of photonic crystal heterostructures composed of two $\mathrm{TiO}_{2}$ inverse opal films with different filling factors. Synthetic Metals 2011, 161 (5-6), 504-507.

[5] Cai, Z.; Teng, J.; Xiong, Z.; Li, Y.; Li, Q.; Lu, X.; Zhao, X. S., Fabrication of TiO2 Binary Inverse Opals without Overlayers via the Sandwich-Vacuum Infiltration of Precursor. Langmuir 2011, 27 (8), 5157-5164.

[6] Reese, C. E.; Guerrero, C. D.; Weissman, J. M.; Lee, K.; Asher, S. A., Synthesis of highly charged, monodisperse polystyrene colloidal particles for the fabrication of photonic crystals. Journal of Colloid and Interface Science 2000, 232 (1), 76-80.

[7] Zhu Wen, Huang Fangting, Yang Runmiao, Zhang Ming, Preparation of Size Controllable Monodispersed Polystyene Microsphere by Dispersion Polymerization. Journal of Materials Sciences and Engineering 2012, 138(4):595-599.

[8] Pang Chengxin, Zhang Lixia, Tan Jian, Ye Zhengmei, Chen Jinhao, The Study of Preparing Nanocrystalline $\mathrm{SnO}_{2}$ 
by Sol-gel Method. Journal of Guangxi Teachers Education University(Natural Science Edition), 2006,23(3):26-40.

[9] P. Jiang, J. F. Bertone, K. S. Hwang, and V. L. Colvin, Single-Crystal Colloidal Multilayers of Controlled Thickness. Chem. Mater. 1999, 11, 2132-2140.

[10] Yan, G.; Zhang, X.; Huang, P.; Wang, L.; Qi, F.; Feng, B., Influence of deposition time on the morphology and optical properties of $\mathrm{SiO} 2-\mathrm{ZnO}$ composite photonic crystals. Chinese Science Bulletin 2011, 56 (6), 562-566. 